\title{
Opioids for chronic noncancer pain
}

\section{To prescribe or not to prescribe-What is the question?}

(6)

Stephen E. Nadeau, MD

Correspondence to

Dr. Nadeau:

snadeau@ufl.edu

\section{ABSTRACT}

The recent American Academy of Neurology position paper by Franklin, "Opioids for chronic noncancer pain," suggests that the benefits of opioid treatment are very likely to be substantially outweighed by the risks and recommends avoidance of doses above 80-120 mg/day morphine equivalent. However, close reading of the primary literature supports a different conclusion: opioids have been shown in randomized controlled trials (RCTs) to be highly effective in the treatment of chronic nonmalignant pain; long-term follow-up studies have shown that this effectiveness can be maintained; and effectiveness has been limited in many clinical trials by failure to take into account high variability in dose requirements, failure to adequately treat depression, and use of suboptimal outcome measures. Frequency of side effects in many RCTs has been inflated by overly rapid dose titration and failure to appreciate the high interindividual variability in side effect profiles. The recent marked increase in incidence of opioid overdose is of grave concern, but there is good reason to believe that it has been somewhat exaggerated. Potential causes of overdose include inadequately treated depression; inadequately treated pain, particularly when compounded by hopelessness; inadvertent overdose; concurrent use of alcohol; and insufficient practitioner expertise. Effective treatment of pain can enable large numbers of patients to lead productive lives and improve quality of life. Effective alleviation of suffering associated with pain falls squarely within the physician's professional obligation. Existing scientific studies provide the basis for many improvements in pain management that can increase effectiveness and reduce risk. Many potentially useful areas of further research can be identified. Neurology ${ }^{\circledR} 2015 ; 85: 646-651$

\section{GLOSSARY}

RCT = randomized controlled trial; VAS = visual analog scale.

The recent American Academy of Neurology position paper by Franklin, "Opioids for chronic noncancer pain," 1 suggests that the benefits of opioid treatment are very likely to be substantially outweighed by the risks (see especially figure 2) and recommends avoidance of doses above $80-120 \mathrm{mg} /$ day morphine equivalent. However, on close reading, the many published treatment studies, most of them randomized controlled trials (RCTs) (see e.g., list by Ballantyne and $S^{2}{ }^{2}$ ), provide a very different picture, indicating that opioids can be highly effective, especially when adequately titrated. The studies demonstrate that opioid effects are well-sustained at stable doses for extended periods and that there are many opportunities for enhancing effectiveness and safety and minimizing side effects, not to mention many issues amenable to further scientific study. The paper implicitly dismisses relief of suffering as a sufficient goal of pain treatment and fails to mention the burden of suffering borne by so many patients with noncancer pain or our responsibility to them as physicians. Patients with chronic noncancer pain report a health-related quality of life as poor as patients dying of cancer. ${ }^{3}$

This presentation of "the other side" of the opioid debate will be solidly evidence-based, with much of the evidence deriving from the primary papers referenced in the review papers cited by Franklin. ${ }^{1}$ However, I will have recourse to my 30 years of clinical experience to illustrate certain

From the Research Service, Malcom Randall VA Medical Center and the Department of Neurology, University of Florida College of Medicine, Gainesville, FL.

Go to Neurology.org for full disclosures. Funding information and disclosures deemed relevant by the author, if any, are provided at the end of the article. 
points, to cite discrepancies between clinical experience and trial results (thus raising questions about study methodology), and to highlight areas that need further study.

Every physician sufficiently experienced in the treatment of chronic noncancer pain knows that opioids, when properly selected and adequately titrated, are effective, no less than for perioperative pain. In fact, the effect is of sufficient magnitude to be classifiable as a "penicillin effect"-the results in a single patient can be, and typically are, sufficiently dramatic to prove the case. While many would view this assertion as preposterous, it finds strong support in the results of RCTs in which participants were pretested to ensure drug tolerability and highly flexible dose titration was used. For example, in an RCT of extendedrelease oxymorphone in 250 patients (mean morphine equivalent daily dose $262 \mathrm{mg}$, range $60-780 \mathrm{mg}$ ), $72 \%$ of patients rated their medication as "very good" or "excellent" and only $10 \%$ withdrew because of side effects. ${ }^{4}$ If this can be accomplished in an RCT, consider what can be achieved with highly individualized and optimized treatment in a clinical setting. RCTs, and in particular phase 3 trials, are and should continue to be the gold standard paradigm for proof of effectiveness of medical interventions. Their particular value derives from the fact that nearly every treatment in medicine has only modest effectiveness, and evidence of that effectiveness may be lost in the noise of interindividual variability and disease fluctuation even as it is susceptible to bias from various sources, hence incorrect imputation of treatment effect. However, medicine is complicated and there is no single formula for answering every question. The enormous power of RCTs notwithstanding, there are certain questions that do not require their use. It did not require an RCT to provide sufficient proof of the effectiveness of penicillin to move it into clinical practice. It did not require RCTs to provide sufficient proof of the effectiveness of opioids to justify their use in the treatment of chronic noncancer pain. Rather, the role of larger trials in this circumstance is to determine generalizability, side effects, and safety; the factors that influence them; and the degree to which analgesia can be stably maintained over time. There continues to be a need for such trials (see Noble et al. ${ }^{5}$ ). Furthermore, as we shall see, the complexities of opioid management of chronic pain pose enormous challenges to RCT design and limit the potential for generalization of RCT results, even as other trial designs may be more effective in addressing key scientific questions. As $\mathrm{Katz}^{6}$ noted: "opioids are not the same as other analgesics, and need to be studied in a manner that reflects their appropriate clinical use."

Chronic pain is one of the most common conditions affecting human beings and causes great suffering. Because of the existing evidence that it is highly treatable, the question arises: Is it ethical for a physician who is knowledgeable in the treatment of pain to withhold opioid treatment? With RCTs, can equipoise be established in a placebocontrolled trial? That is, can a population of patients with chronic pain be defined in which it is truly unknown whether treatment with opioids will be more effective than treatment with placebo? As it turns out, investigators have largely "finessed" this issue by the ethically essential but methodologically deleterious device of allowing patients to drop out of trials. Dropout rates may be as high as $75 \%$ in placebo arms. ${ }^{4}$

OPIOID TREATMENT Are there questions about opioid treatment of chronic noncancer pain that require scientific study? Most assuredly:

1. Variability in dose requirements. We implicitly accept the need for dose titration in the use of postoperative patient-controlled analgesia. Published studies report a 15 -fold variability in dose requirements for treatment of postoperative pain, that is, in opioid-naive patients ${ }^{7-10}$ (see also Katz ${ }^{6}$. My own experience in the treatment of chronic noncancer pain is congruent with this. For one patient, $180 \mathrm{mg}$ of oxycodone may be just sufficient and without significant side effects, whereas for another $10 \mathrm{mg}$ may be effective but poorly tolerated. This variability is reflected in RCTs and open-label follow-up studies of treatment of chronic noncancer pain that permitted liberal titration (see studies in Ballantyne and Shin ${ }^{2}$ ).

Failure to appreciate variability in dose requirements has 3 major consequences:

a. To the extent that doses have not been adequately titrated in RCTs of opioids, demonstration of only modest effectiveness likely 
reflected inadequate dose. RCTs have consistently shown benefits, often quite substantial, from opioid treatment, ${ }^{2,11}$ but doses have generally been no more than moderate ( $\leq 180 \mathrm{mg} /$ day morphine equivalent). The short duration of many of these trials limited the opportunity for titration to optimal doses, and when titration was done, it was often very rapid, thereby increasing the frequency of side effects.

b. Titration of opioids, even in the modest ranges usually reported, in patients who are poorly tolerant of opioids in general or of particular opioids (see 2 below) may contribute to the high frequency of side effects often reported in these trials. ${ }^{11}$

c. In clinical practice, patients reporting the need for higher doses are widely viewed as drug seeking, drug dependent, or drug addicted and are often mistreated. However, why would a patient still in intolerable pain not ask for higher doses or a more effective alternative treatment? (See also Weissman and Haddox. ${ }^{12}$ )

The molecular basis for variability in dose requirements has been related to splice variants and allelic variations in the opioid receptor, mu 1 (OPRM1) gene. ${ }^{13}$ How does one determine in clinical practice whether it is safe to titrate further in the face of persistent patient suffering? How can one be certain that higher doses of opioids in a particular patient will alleviate suffering, thereby justifying the incursion of some risk? My own experience is that what might be termed pain of somatic origin (pain that is not neuritic or neuropathic) is nearly always treatable - experience validated by the clinical trial evidence already reviewed. On the other hand, psychic pain related to psychiatric disease (most often depression but not always easily characterized) is much more problematic. How does one tell the difference? Failure to improve analgesia with major increments in analgesic dosage should probably raise this concern.

2. Side effects. A patient with cognitive or neurologic side effects attributable to opioids, whether these are observed by the physician or family members, is clearly receiving excessive dosage. However, there may be other equally important but more subtle signs of excessive dosage. Is excessive dosage to be blamed in the patient who becomes intermittently intoxicated because of a tendency to forget when she has taken her medications, or does this simply reflect a disorder of concentration or hippocampal encoding of other cause? How would one know? Is a patient on a chronic opioid regimen who feels safe to drive truly safe to drive? (See Chou et al. ${ }^{14}$ )
3. Opioid equivalence. The varying potency of various opioids is well-recognized. However, it is not well-recognized that different opioids may have major, idiosyncratic, qualitative differences in their side effect profiles, ${ }^{15,16}$ likely genetic in origin. Dysphoria, euphoria, nausea, vomiting, pruritus, increased sweating, dizziness/imbalance, cognitive impairment, and sleepiness vary from drug to drug in any given patient and usually can be eliminated simply by a drug change. Constipation, the one nearly ubiquitous side effect, is, in my experience, almost always easily treatable with osmotic cathartic regimens that include twice-daily docusate and up to twice-daily polyethylene glycol. Clinical trials testing the effectiveness of variously titrated osmotic regimens in patients chronically taking opioids are lacking.

4. Depression. My own experience is that depression, most often manifested as anhedonia, is very common in chronic noncancer pain populations and is often difficult to treat. Effective treatment of depression (e.g., with well-titrated selective serotonin reuptake inhibitors, possibly in combination with bupropion and mood- stabilizing anticonvulsants such as valproate or lamotrigine, together with counseling) can have as much analgesic effect as opioids, however titrated. In addition, effective treatment of pain is nearly impossible in the context of persistent depression (see also Jamison et al. ${ }^{17}$ ). However, depression is scarcely even mentioned in opioid treatment studies. Studies of opioid overdosing report a depression frequency of $12 \%-27 \%$ even as they report a strong correlation between drug-related encounters or overdoses and depression. ${ }^{18,19}$ This suggests that depression, arguably the single most important comorbidity in patients with chronic pain, is vastly underdiagnosed and that antidepressant treatment is vastly underused in the treatment of chronic noncancer pain.

5. Sleep. Sleep disturbances occur in $50 \%-70 \%$ of patients with chronic pain. ${ }^{20,21}$ To what extent does poor sleep and lying awake all night in pain degrade overall pain control? To what extent would assurance of good sleep enable reduction in opioid dose requirements? My experience is that good sleep can nearly always be achieved by nonopioid measures, e.g., optimization of sleep hygiene, treatment of restless legs syndrome/ periodic limb movements of sleep with dopaminergic agents, treatment of prolonged latency with titrated benzodiazepines (conceivably quetiapine), and treatment of poorly sustained sleep with titrated trazodone. To what extent can a nocturnal hiatus in opioid dosing mitigate tolerance and dependence? 
6. Dose regimens. Drug tolerance and drug dependence are common concerns in the opioid treatment of chronic pain of any cause and rightly so, particularly in the case of tolerance, as the development of tolerance will lead to steady deterioration in analgesia and potentially propel further dose titration. However, extended clinical trials of sustained-release treatment (e.g., transdermal fentanyl and oxycodone controlled-release) suggest that tolerance is very modest and rarely of clinical importance in this population. ${ }^{22-25}$ To what extent is tolerance a product of biology rather than treatment regimen?

7. Endpoints. The standard primary outcome measure in RCTs of opioid effectiveness is a visual analog scale (VAS) of pain intensity. Is this the best measure? The VAS, unlike the more complex McGill Pain Questionnaire, demonstrated almost no correlation with a more objective measure of pain intensity. ${ }^{26}$ VAS anchor points are likely susceptible to drift over time and with treatment in ways that will tend to reduce changes observed. This has not been sufficiently studied. ${ }^{27}$ My own experience is that a typical patient may continue to rate his or her pain as severe regardless of the treatment. However, when the question is put another way (e.g., how well are you controlling your pain with your current regimen?), the answer is typically very different: "Oh doc, I'm doing fine. I don't want to change a thing." On the other hand, a decision to increase dosage typically requires an extended exploration of analgesic adequacy, including consideration of the strong possibility that decline in pain control may reflect recurrent depression and reassessment of cognitive and neurologic side effects.

8. Nonpharmacologic approaches. To what extent can nonpharmacologic approaches to pain management reduce opioid doses needed to achieve satisfactory pain control? Should magnitude of reduction in opioid dosage required be the standard primary outcome measure in trials of adjuvant treatments?

OVERDOSE RISKS Franklin ${ }^{1}$ rightly raises concern over the recent rise in deaths due to opioid overdose. The crux of the problem is that higher prescribed amounts of opioids, even if still inadequate, also provide the means for overdose death. The increase in opioidassociated deaths is of sufficient magnitude that we absolutely must find measures to stem it, but any discussion of this topic that fails to take into account the many patients who are able to work, be creative, and enjoy quality personal and family life thanks to effective treatment of pain is bound to be unbalanced.
Reduction of the incidence of opioid overdose is a complicated challenge that demands sophisticated scientific inquiry. The following are key issues:

1. Validity of reports of overdose risk. Studies of opioid-related deaths that rely on demonstration of sufficient tissue opioid concentrations to define causality do demonstrate increased risk of overdose death with high opioid dosage ( $\geq 200 \mathrm{mg}$ / day morphine equivalent, odds ratio 2.88). ${ }^{28}$ However, this is considerably lower than in studies in which overdose death was determined clinically (e.g., hazard ratio $7.18^{29}$ ). This suggests that many deaths are falsely attributed to opioids, particularly when patients are receiving "high-dose" regimens. The same problem may affect studies of opioid overdoses in general. ${ }^{18}$ To what extent are serious underlying causes of somnolence overlooked, or diagnoses delayed, in patients taking opioids?

2. Inadequate dosing. Because of current widely held beliefs about opioids, underdosing with opioids is likely more the rule than the exception. Compounding this is the highly prevalent abuse that patients experience from physicians, other medical care providers, pharmacists, and even family members-borne of the common perception that any person on a "high-dose" opiate regimen must be an addict-and the humiliation patients feel. In this context, many patients develop what might be characterized as an Armageddon outlook: I don't want to die but I am so tired of hurting and there is no hope, so if I take more of this drug than I am supposed to at least it will relieve my suffering, and if I die, well, it will mean lasting relief. Suicide rates are elevated in chronic pain populations, particularly in the context of a sense of hopelessness. ${ }^{30,31}$

3. Depression. I have already alluded to the evidence that depression is likely to be vastly underdiagnosed in patients with chronic pain, much less adequately treated-hence inadequate analgesia and likely potentiation of an Armageddon outlook.

4. Inadvertent overdose. To what extent do overdoses occur because patients have forgotten that they have recently dosed? To what extent is this a consequence of opioid effects on concentration and memory? Should drug-dispensing devices that would preclude repeat dosing within certain time spans be used in patients taking opioids to treat chronic pain?

5. Sleep. To what extent does daytime impairment in concentration commonly observed with qualitative disorders of sleep, such as restless legs syndrome/periodic limb movements of sleep and obstructive sleep apnea, conduce to inadvertent overdose? 
6. Alcohol. The potentially deadly interactions of alcohol and opioids have been extensively documented. How often does alcohol play a role in opioid overdoses, whether because of patient ignorance about the deadliness of the interaction, patient use of alcohol as an adjuvant to pain relief, casual societal attitudes about alcohol consumption, or conscious acting out of an Armageddon outlook?

7. Practitioner expertise. To what extent are opioid overdoses related to lack of skill or bad practices by the prescriber? Since we do not even have a scientifically informed consensus on what represents good or bad practice, it seems plausible that

\section{Comment: \\ Prescription opioids-Misused or misunderstood?}

Widespread use of opioids for chronic noncancer pain has resulted in an epidemic of misuse; prescribed opioids are currently responsible for more deaths in the United States than heroin and cocaine combined. An American Academy of Neurology (AAN) position paper questions the long-term efficacy of opioids for chronic noncancer pain, calls attention to the poor safety profile of opioids, urges regulatory guidelines in their use, and calls for more research on chronic pain management. ${ }^{1}$

Dr. Nadeau challenges these conclusions, declaring, "The paper implicitly dismisses relief of suffering as a sufficient goal of pain treatment...." ${ }^{2} \mathrm{He}$ asserts, moreover, that the increased incidence of opioid overdose "has been somewhat exaggerated." Dr. Nadeau's major message-that studies of opioid benefit and risk have not taken into account "interindividual variability"-addresses a legitimate concern, namely, that regulatory responses to the prescription opioid epidemic could result in a one-size-fits-all straitjacket. However, he misleadingly suggests that the AAN favors such regulatory rigidity. In fact, the AAN paper declares, "The core of this Guideline is a recommendation for a prescribing provider to seek consultation if a patient reaches $120 \mathrm{mg} / \mathrm{d}$ MED and if pain and function have not substantially improved."

Opining that the efficacy of opioids is "well-sustained at stable doses for extended periods," Dr. Nadeau cites a literature review by Ballantyne and Shin. In fact, those authors emphasized the lack of evidence for "long-term effectiveness." Not cited by Dr. Nadeau is a 2013 Cochrane Review that concluded, "There are no placebo-controlled randomized trials supporting the effectiveness and safety of long-term opioid therapy for treatment of chronic low-back pain." 3 Nadeau likens the analgesic efficacy of opioids to a "penicillin effect," obviating the need for controlled studies. The issue here, however, is chronic pain. Is the benefit of long-term opioids for unremitting low back pain really as obvious as the benefit of penicillin in treating bacterial meningitis?

Dr. Nadeau appropriately calls for nuanced approaches in treating chronic pain. Clinicians should be wary, however, when he claims that the long-term efficacy of opioid treatment for chronic pain is established and that the risks of unfettered opioid use are exaggerated.

1. Franklin GM; American Academy of Neurology. Opioids for chronic noncancer pain: a position paper of the American Academy of Neurology. Neurology 2014;83:12771284.

2. Nadeau SE. Opioids for chronic noncancer pain: to prescribe or not to prescribewhat is the question? Neurology 2015;85:646-651.

3. Chaparro LE, Furlan AD, Deshpande A, Mailis-Gagnon A, Atlas S, Turk DC. Opioids compared to placebo or other treatments for chronic low-back pain. Cochrane Database Syst Rev 2013;8:CD004959. doi: 10.1002/14651858.

John C.M. Brust, MD

From the Department of Neurology, Columbia University College of Physicians \& Surgeons, New York Neurological Institute, New York, NY.

Study funding: No targeted funding reported.

Disclosure: The author reports no disclosures relevant to the manuscript. Go to Neurology.org for full disclosures. prescribing practices may be a major contributor to opioid overdose.

CONCLUSION No component of medical practice hews more to the spirit of Hippocrates and Maimonides than the treatment of pain. Opioids are often an essential component of this treatment, but we have discovered that using opioids in a way that consistently alleviates pain and suffering without incurring harm is a complex challenge that, left to the chaotic order of medical practice, has not been adequately met. Ideas on how to address this challenge abound, and if we can avoid the appeal of simplistic solutions and pursue the necessary scientific inquiry, there is no doubt that we can meet it.

Pain and suffering are products of the CNS. Thus, neurologists are optimally qualified not only to manage it but also to take a lead in developing processes of pain management that will maximize relief of suffering and minimize risk of overdose by taking into account the complexity of opioid management.

\section{STUDY FUNDING}

This work was supported by resources provided by the North Florida/ South Georgia Veterans Health System, Gainesville, FL. The contents of this manuscript do not represent the views of the US Department of Veterans Affairs or the United States Government.

\section{DISCLOSURE}

S. Nadeau receives royalties from MIT Press for the book The Neural Architecture of Grammar. He receives research support through Department of Veterans Affairs grants B9252-C and RX001183-01A1. He has served as a member of the data safety monitoring board for the Interdisciplinary Study of Arm Rehabilitation after Stroke (ICARE) Trial (NIH/National Institute of Neurological Disorders and Stroke) (NCT00871715). Go to Neurology.org for full disclosures.

Received January 10, 2015. Accepted in final form March 31, 2015.

\section{REFERENCES}

1. Franklin GM; American Academy of Neurology. Opioids for chronic noncancer pain.:a position paper of the American Academy of Neurology. Neurology 2014;83:1277-1284.

2. Ballantyne JC, Shin NS. Efficacy of opioids for chronic pain. Clin J Pain 2008;24:469-478.

3. Fredheim OM, Kaasa S, Fayers P, Saltnes T, Jordhøy M, Borchgrevink $\mathrm{P}$. Chronic non-malignant pain patients report as poor health-related quality of life as palliative cancer patients. Acta Anaesthesiol Scand 2008;52:143-148.

4. Hale ME, Ahdieh H, Ma T, Rauck R; Oxymorphone ER Study Group 1. Efficacy and safety of OPANA ER (oxymorphone extended release) for relief of moderate to severe low back pain in opioid-experienced patients: a 12-week, randomized, double-blind, placebo-controlled study. J Pain 2007;8:175-184.

5. Noble M, Treadwell JR, Tregear SJ, et al. Long-term opioid management for chronic noncancer pain. Cochrane Database Syst Rev 2010:CD006605.

6. Katz N. Methodological issues in clinical trials of opioids for chronic pain. Neurology 2005;65(suppl 4):S32-S49.

7. Beeton AG, Upton PM, Shipton EA. The case for patientcontrolled analgesia. S Afr J Surg 1992;30:5-6. 
8. Cepeda MS, Carr DB. Women experience more pain and require more morphine than men to achieve a similar degree of analgesia. Anesth Analg 2003;97:1464-1468.

9. Leitao MM, Malhotra V, Briscoe G, et al. Postoperative pain medication requirements in patients undergoing computer-assisted ("robotic") and standard laparoscopic procedures for newly diagnosed endometrial cancer. Ann Surg Oncol 2013;20:3561-3567.

10. Okutomi T, Saito M, Mochizuki J, Amano K, Hoka S. A double-blind randomized controlled trial of patientcontrolled epidural analgesia with or without a background infusion following initial spinal analgesia for labor pain. Int J Obstet Anesth 2009;18:28-32.

11. Furlan AD, Chaparro LE, Irvin E, Mailis-Gagnon A. A comparison between enriched and nonenriched enrollment randomized withdrawal trials of opioids for chronic noncancer pain. Pain Res Manag 2011;16:337-351.

12. Weissman DE, Haddox JD. Opioid pseudoaddiction-an iatrogenic syndrome. Pain 1989;36:363-366.

13. Pasternak GW. Opioids and their receptors: are we there yet? Neuropharmacology 2014;76:198-203.

14. Chou R, Ballantyne JC, Fanciullo GJ, Fine PG, Miaskowski C. Research gaps on use of opioids for chronic noncancer pain: findings from a review of the evidence for an American Pain Society and American Academy of Pain Medicine clinical practice guideline. J Pain 2009;10:147-159.

15. Quang-Cantagrel N, Wallace MS, Magnuson SK. Opioid substitution to improve the effectiveness of chronic noncancer pain control: a chart review. Anesth Analg 2000;90: 933-937.

16. Cherny N, Ripamonti C, Pereira J, et al. Strategies to manage the adverse effects of oral morphine: an evidence-based report. J Clin Oncol 2001;19:2542-2554.

17. Jamison RN, Edwards RR, Liu X, et al. Relationship of negative affect and outcome of an opioid therapy trial among low back pain patients. Pain Pract 2012;13: 173-181.

18. Dunn KM, Saunders KW, Rutter CM, et al. Opioid prescriptions for chronic pain and overdose. Ann Intern Med 2010;152:85-92.
19. Braden JB, Russo J, Fan M-Y, et al. Emergency department visits among recipients of chronic opioid therapy. Arch Intern Med 2010;170:1425-1432.

20. Cohen MJM, Menefee LA, Doghramji K, Anderson WR, Frank ED. Sleep in chronic pain: problems and treatments. Int Rev Psychiatry 2000;12:115-127.

21. Pilowsky I, Crettenden I, Townley M. Sleep disturbance in pain clinic patients. Pain 1985;23:27-33.

22. Milligan K, Lanteri-Minet M, Borchert K, et al. Evaluation of long-term efficacy and safety of transdermal fentanyl in the treatment of chronic noncancer pain. J Pain 2001;2:197-204.

23. Mystakidou K, Parpa E, Tsilika E, et al. Long-term management of noncancer pain with transdermal therapeutic system-fentanyl. J Pain 2003;4:298-306.

24. Portenoy RK, Farrar JT, Backonja M-M, et al. Long-term use of controlled-release oxycodone for noncancer pain: results of a 3-year registry study. Clin J Pain 2007;23:287-299.

25. Roth SH, Fleischmann RM, Burch FX, et al. Around-theclock, controlled-release oxycodone therapy for osteoarthritisrelated pain. Arch Intern Med 2000;160:853-860.

26. Kim J, Lee KS, Kong SW, et al. Correlations between electrically quantified pain degree, subjectively assessed visual analogue scale, and the McGill Pain Questionnaire: a pilot study. Ann Rehabil Med 2014;38:665-672.

27. Breivik H, Borchgrevink PC, Allen SM, et al. Assessment of pain. Br J Anaesth 2008;101:17-24.

28. Gomes T, Mamdani MM, Dhalla IA, Paterson JM, Juurlink DN. Opioid dose and drug-related mortality in patients with nonmalignant pain. Arch Intern Med 2011; 171:686-691.

29. Bohnert ASB, Valenstein M, Bair MJ, et al. Association between opioid prescribing patterns and opioid overdoserelated deaths. JAMA 2011;305:1315-1321.

30. Fishbain DA, Goldberg M, Rosomoff RS, Rosomoff $\mathrm{H}$. Completed suicide in chronic pain. Clin J Pain 1991;7: 29-36.

31. Tang NKY, Crane C. Suicidality in chronic pain: a review of the prevalence, risk factors, and psychological links. Psychol Med 2006;36:575-586. 